\title{
LncRNA TUG1 promotes the development of osteosarcoma through RUNX2
}

\author{
KUNKUN SHENG and YAN LI \\ Department of Orthopedics, The Second Affiliated Hospital, \\ Zhejiang University School of Medicine, Hangzhou, Zhejiang 310009, P.R. China
}

Received July 1, 2018; Accepted June 27, 2019

DOI: $10.3892 /$ etm.2019.7880

\begin{abstract}
The lncRNA taurine-upregulated gene 1 (TUG1) is known to serve a role as an oncogene in the development of a number of human malignancies. However, the functionality of TUG1 in osteosarcoma remains poorly characterized. Therefore, the aim of the present study was to explore the role of TUG1 in osteosarcoma. TUG1 expression in tumor tissues, adjacent healthy tissues and plasma from 40 osteosarcoma patients and 40 healthy controls was detected using reverse transcription-quantitative PCR. Receiver operating characteristic curves were used to analyze the diagnostic value of TUG1 for osteosarcoma while the prognostic value of TUG1 for osteosarcoma was analyzed using the Kaplan-Meier method. TUG1 expression vectors and siRNAs were transfected into MG-63 and U2OS osteosarcoma cell lines, and the effects on osteosarcoma cell viability, migration and invasion were tested using Cell Counting kit-8 and Transwell assays. The effects of TUG1 overexpression on runt-related transcription factor 2 (RUNX2) expression were also detected using western blotting. TUG1 expression was found to be significantly higher in osteosarcoma tissues compared with adjacent healthy tissues, and in the plasma of osteosarcoma patients compared with healthy controls. TUG1 expression also exhibited significant diagnostic and prognostic value for osteosarcoma. TUG1 overexpression and knockdown respectively increased and reducedosteosarcoma cell viability, migration and invasion. In addition, TUG1 overexpression upregulated RUNX2 expression. These results suggest that lncRNA TUG1 may promote the development of osteosarcoma by modulating RUNX2 and TUG1 expression, which can serve as prognostic and diagnostic markers for this malignancy.
\end{abstract}

Correspondence to: $\mathrm{Dr}$ Kunkun Sheng, Department of Orthopedics, The Second Affiliated Hospital, Zhejiang University School of Medicine, 88 Jiefang Road, Hangzhou, Zhejiang 310009, P.R. China

E-mail: shengkunkun@zju.edu.cn

Key words: long noncoding RNA, taurine up-regulated gene 1, osteosarcoma, runt-related transcription factor 2, reverse transcription-quantitative PCR, small interfering RNA silencing

\section{Introduction}

Osteosarcoma is the most common type of primary malignant tumor in the bone tissue (1). Whilst most younger patients (10-30 years old) suffer from primary conventional osteosarcoma, elderly patients ( $>65$ years) are more likely have secondary osteosarcoma (2). Osteosarcoma mainly originates from the long bones, but other bones may also be affected by the disease (3). It is estimated that between 0.8 and 11 individuals per 100,000 are affected by this disease (4), with 1,000 new cases of osteosarcoma reported in the United States every year (2). Despite the low incidence rate, osteosarcoma is considered to be an important cause of cancer-related mortality (3). Patients with osteosarcoma are usually treated with aggressive adjuvant chemotherapy and surgical resection $(3,4)$. With the development of novel treatment strategies, treatment outcomes of osteosarcoma have improved significantly during the past decades (1). However, the prognosis for osteosarcoma patients remains fairly poor, particularly for patients with metastatic or recurrent osteosarcoma, for whom the 5-year survival rate is $<25 \%$ (3).

The development of osteosarcoma is a complex process that involves a number of internal and external factors. High levels of genomic instability are common in patients with osteosarcoma, and activation of oncogenes and mutations of tumor suppressor genes have been found to be at least partially responsible for the occurrence of this disease (1). The role of noncoding RNAs has been extensively studied in a variety of pathophysiological processes (5). Long non-coding RNA (lncRNA) is a group of noncoding RNAs that are $>200$ nucleotides in length (6), and significantly longer than miRNA, siRNA and other short noncoding RNAs. One such lncRNA, taurine-upregulated gene 1 (TUG1), has been demonstrated to serve different functions in different types of malignancies $(7,8)$. In glioma, TUG1 acts as a tumor suppressor gene by promoting cancer cell apoptosis (7), whereas it has been reported to be upregulated in esophageal squamous cell carcinoma, where it promotes cancer cell proliferation and migration (8). In addition, TUG1 has been reported to be upregulated in osteosarcoma, with this upregulation being closely correlated with poor prognosis of osteosarcoma patients (9). However, the functionality of this remains unclear.

In the present study, the expression of TUG1 in the tumor tissues and adjacent healthy tissues of osteosarcoma patients, 
and in plasma samples from osteosarcoma patients and healthy controls was analyzed. The diagnostic and prognostic values of TUG1 for osteosarcoma were analyzed. In addition, the effects of TUG1 overexpression and silencing on osteosarcoma cell viability, migration and invasion were tested. The effects of TUG1 overexpression on proteins associated with the epithelial-mesenchymal transition (EMT) pathway were also explored.

\section{Materials and methods}

Patients. A total of 40 patients with osteosarcoma (22 males and 18 females; age range, 11-78 years; average age, $41 \pm 11.1$ years) were selected in the 2nd Affiliated Hospital of Zhejiang University School of Medicine (Zhejiang, China) from January 2009 to January 2011. Inclusion criteria: i) All patients were diagnosed by pathological and imaging examination; ii) newly diagnosed cases. Exclusion criteria: i) Recurrent patients; ii) patients who have been treated; iii) patients with multiple newly diagnosed clinical disorders. Distant metastasis was detected in 26 patients. All patients received surgical resections, with tumor tissues and adjacent healthy tissues within the region $0.5 \mathrm{~cm}$ from the tumor boundary being collected during surgery. Within the same timeframe, 40 healthy individuals (20 males and 20 females; age range, 16-72 years; average age, $40 \pm 9.8$ years) were selected as controls. Blood $(5 \mathrm{ml})$ was extracted from each patient and control before therapies under fasting conditions. Blood was centrifuged in EDTA tubes at room temperature for $10 \mathrm{~min}$ at $1,200 \times \mathrm{g}$ to obtain plasma. There were no significant differences in age, sex and other basic information between the two groups. This study was approved by the ethics committee of The 2nd Affiliated Hospital of Zhejiang University School of Medicine, and all patients provided signed informed consent. Follow-up was performed for 60 months to monitor the survival of the patients.

Cell lines and cell culture. Human normal bone cell line hFOB and osteosarcoma cell lines MG-63 and U2OS were purchased from American Type Culture Collection (ATCC). According to the supplier's protocol, hFOB cells were cultivated in a mixture of $45 \%$ Dulbecco's modified Eagle's medium, 45\% Ham's F12 Medium and 10\% fetal bovine serum (all Sigma-Aldrich; Merck KGaA), U2OS cells were cultured using ATCC-formulated McCoy's 5A Medium (cat no. 30-2007; ATCC) containing 10\% fetal bovine serum, whilst MG-63 cells were cultured with Eagle's Minimum Essential Medium (cat no. 30-2003; ATCC) containing 10\% heat-inactivated fetal bovine serum (Sigma-Aldrich; Merck KGaA) maintained in a humidified atmosphere at $37^{\circ} \mathrm{C}$ under $5 \% \mathrm{CO}_{2}$. Cells were harvested upon reaching the logarithmic growth phase for subsequent experiments.

Cell transfection. Silencer ${ }^{\mathrm{TM}}$ Select Negative Control No. 1 siRNA (5'-UUCGAGAGAUGCACGGAAAU-3'; cat. no. 4390843) and TUG1 siRNA (5'-GGGAUAUAGCCA GAGAACAAUUCU-3'; cat. no. 1299001; both Thermo Fisher Scientific, Inc.) were used to establish TUG1-silenced cell lines. A TUG1 overexpression vector was established using pIRSE2-EGFP vector backbone and empty pIRSE2-EGFP vector backbone vectors were also used (Clontech Laboratories, Inc.). The vector construction service was provided by Sangon Biotech Co., Ltd. Cells ( $10^{6}$ in $10 \mathrm{ml}$ media; a different medium for different cell lines) were cultured overnight to reach $80-90 \%$ confluence, and transfection (40 nM siRNA or $10 \mathrm{nM}$ vector) was performed using Lipofectamine ${ }^{\circledR} 2000$ reagent according to the manufacturer's protocol (Invitrogen; Thermo Fisher Scientific, Inc.). Following experiments were performed using cells harvested at $24 \mathrm{~h}$ post-transfection.

Reverse transcription-quantitative PCR (RT-qPCR). TRIzol reagent (Invitrogen; Thermo Fisher Scientific, Inc.) was used to extract total RNA from tissues, plasma samples and cell lines according to the manufacturer's protocol. RNA quality was assessed using the NanoDrop ${ }^{\mathrm{TM}} 2000$ Spectrophotometer (Thermo Fisher Scientific, Inc.) to determine the absorbance values at $260 \mathrm{~nm}$ (A260) and A280. Only RNA samples with A260/A280 ratios of between 1.8 and 2.0 were used for RT to synthesize cDNA. RT was carried out using RevertAid RT Reverse Transcription kit (Thermo Fisher Scientific, Inc.) with the following conditions: $55^{\circ} \mathrm{C}$ for $30 \mathrm{~min}$ and $80^{\circ} \mathrm{C}$ for $10 \mathrm{~min}$. qPCR systems were prepared using SYBR ${ }^{\circledR}$ Green Real-Time PCR Master mix according to the manufacturer's protocol (Thermo Fisher Scientific, Inc.). The following primers were used for the qPCR step: TUG1 forward, 5'-TTGTCACGT CCACCGGACCTG-3' and reverse, 5'-CACAAATTCCCA TCATTCCC-3'; runt-related transcription factor 2 (RUNX2) forward, 5'-CCTGAACTCTGCACCAAGTC-3' and reverse, 5'-GAGGTGGCAGTGTCATCATC-3'; and $\beta$-actin forward, 5'-GACCTCTATGCCAACACAGT-3' and reverse, 5'-AGT ACTTGCGCTCAGGAGGA-3'. CFX96 Touch ${ }^{\text {TM }}$ Real-Time PCR Detection System (Bio-Rad Laboratories, Inc.) was used to perform qPCR. The thermocycling conditions for $\mathrm{qPCR}$ were: Initial denaturation at $95^{\circ} \mathrm{C}$ for $30 \mathrm{sec}$, followed by 40 cycles of $95^{\circ} \mathrm{C}$ for $12 \mathrm{sec}$ and $60^{\circ} \mathrm{C}$ for $30 \mathrm{sec}$. Data were processed using the $2^{-\Delta \Delta \mathrm{Cq}}$ method (10). Relative expression levels of TUG1 were normalized to those of the endogenous control $\beta$-actin.

Cell viability assay. Cells were seeded into 96-well plates at $6 \times 10^{3}$ cells/well. After incubation for 3-5 h to allow cell adhesion, $100 \mu \mathrm{l}$ aforementioned media was added. A total of $10 \mu \mathrm{l}$ Cell Counting Kit-8 (CCK-8; Sigma-Aldrich; Merck $\mathrm{KGaA}$ ) reagent was added after $24,48,72$ and $96 \mathrm{~h}$ of incubation. Following incubation for a further $3 \mathrm{~h}$, optical density values at $450 \mathrm{~nm}$ were measured for each well using a microplate reader.

Cell migration and invasion assay. Transwell ${ }^{\circledR}$ cell migration assay (BD Biosciences) was performed. Briefly, the upper chamber was seeded with $3 \times 10^{4}$ cells diluted in serum-free aforementioned media, while RPMI-1640 medium (Thermo Fisher Scientific, Inc.) containing 20\% fetal calf serum (Sigma-Aldrich; Merck KGaA) was used to fill the lower chamber. The membranes of the Transwell chambers were collected after $24 \mathrm{~h}$ incubation, membranes were fixed in $70 \%$ ethanol for $1 \mathrm{~min}$ at room temperature, followed by staining with $0.5 \%$ crystal violet (Sigma-Aldrich; Merck KGaA) for $20 \mathrm{~min}$ at room temperature. Stained cells were counted under a light microscope. Five visual fields were selected to count 


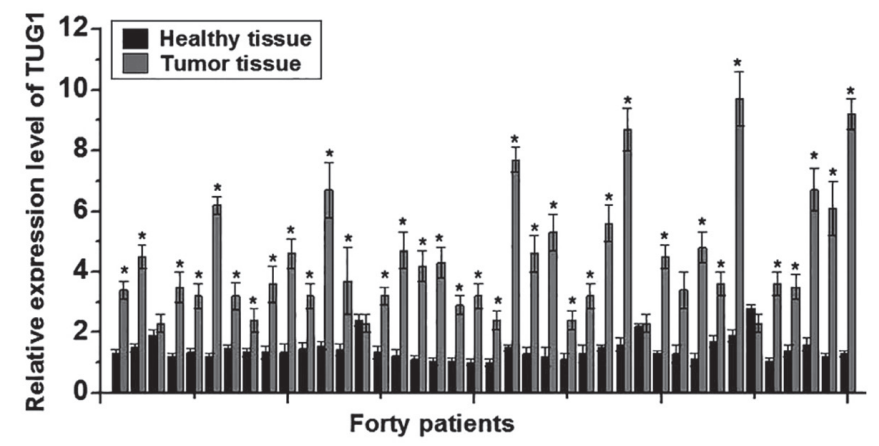

Figure 1. Relative expression of long non-coding RNA TUG1 in osteosarcoma tissues and adjacent healthy tissues. Each experiment was repeated three times. ${ }^{\mathrm{P}}<0.05$ vs. healthy tissue. TUG1, taurine upregulated gene 1.

cells. In cell invasion assays, the upper chamber was first pre-coated with $200 \mathrm{mg} / \mathrm{nl}$ Matrigel ${ }^{\circledR}$ (cat. no. 356234; EMD Millipore; Merck KGaA) for $12 \mathrm{~h}$ at $37^{\circ} \mathrm{C}$, and all other procedures were identical to those of the migration assays. Cell migration and invasion rates were normalized to cell viability rates at $24 \mathrm{~h}$ using the CCK- 8 assay.

Western blot analysis. Total protein extraction was performed using RIPA solution (Thermo Fisher Scientific, Inc.), and protein concentration was assessed using a bicinchoninic acid assay. A total of $30 \mu \mathrm{g}$ protein from each sample was separated by $10 \%$ SDS-PAGE and then transferred to PVDF membranes. Blocking was performed by incubation with $5 \%$ skimmed milk in PBS at room temperature for $2 \mathrm{~h}$. Following washing, membranes were incubated with rabbit polyclonal anti-RUNX2 (1:1,000; cat. no. ab23981) or rabbit polyclonal anti-GAPDH (1:1,000; cat. no. ab9485) primary antibodies (all Abcam) overnight at $4^{\circ} \mathrm{C}$. Membranes were washed and incubated with goat anti-rabbit IgG secondary antibody conjugated to horseradish peroxidase (1:1,000; cat. no. MBS435036; MyBioSource, Inc.) at room temperature for $3 \mathrm{~h}$. Following another round of washing, ECL (Sigma-Aldrich; Merck $\mathrm{KGaA}$ ) was added to visualize the protein bands. Signals were then scanned using the MYECL ${ }^{\mathrm{TM}}$ Imager (Thermo Fisher Scientific, Inc.). Relative expression levels of each protein were normalized to those of the endogenous control $\beta$-actin using Image J v1.48 software (National Institutes of Health).

Statistical analysis. SPSS 19.0 software (IBM Corp.) was used for all statistical analyses. Normally distributed data are presented as the mean \pm standard deviation. Comparisons between two groups were performed using unpaired t-test, comparisons between tumor and healthy tissues were performed using paired test, and comparisons among multiple groups were performed using one-way ANOVA followed by Tukey's test. Patients with osteosarcoma were divided into two groups according to the median expression level of TUG1 mRNA in plasma (3.98). Survival curves were plotted using the Kaplan-Meier method and compared using the log-rank test. Non-normally distributed data were analyzed using the Mann-Whitney U test. The associations between clinicopathological features and TUG1 expression levels in plasma were analyzed using Chi-square test. Receiver operating characteristic (ROC) curve analysis was performed to analyze the
Table I. Association between clinicopathological features and TUG1 expression levels in plasma.

\begin{tabular}{lrrrr}
\hline & \multicolumn{3}{c}{ TUG1 expression } & \\
Clinicopathological features & $\mathrm{N}$ & High & Low & P-value \\
\hline Sex & & & & 0.53 \\
Male & 22 & 12 & 10 & \\
Female & 18 & 8 & 10 & \\
Age (years) & & & & 0.11 \\
$>40$ & 21 & 13 & 8 & \\
$\leq 40$ & 19 & 7 & 12 & \\
Drinking & & & & 0.17 \\
Yes & 28 & 16 & 12 & \\
No & 12 & 4 & 8 & \\
Smoking & & & & 1.00 \\
Yes & 24 & 12 & 12 & \\
No & 16 & 8 & 8 & \\
Metastasis & & & & $<0.001$ \\
Yes & 26 & 19 & 7 & \\
No & 14 & 1 & 13 & \\
\hline
\end{tabular}

TUG1, taurine-upregulated gene 1 .

diagnostic value of TUG1 expression for osteosarcoma. $\mathrm{P}<0.05$ was considered to indicate a statistically significant difference.

\section{Results}

Relative expression levels of IncRNA TUG1 in osteosarcoma and adjacent healthy tissues. In this study, RT-qPCR was performed to detect the expression of TUG1 lncRNA in the cancer tissues and adjacent healthy tissues of 40 patients with osteosarcoma. The expression levels of TUG1 were significantly higher in cancer tissues compared with adjacent healthy tissues in $37 / 40$ patients $(\mathrm{P}<0.05$; Fig. 1$)$, suggesting that lncRNA TUG1 is likely to be involved in the development of osteosarcoma.

Relative expression levels of IncRNA TUG1 in the plasma of osteosarcoma patients and healthy controls. Expression levels of TUG1 in the plasma samples of 40 osteosarcoma patients and 40 healthy controls were also detected using RT-qPCR. TUG1 levels in the plasma of osteosarcoma patients were significantly higher compared with those in healthy controls ( $\mathrm{P}<0.05$; Fig. 2A). ROC curve analysis was also performed to analyze the diagnostic value of TUG1 expression for osteosarcoma. The area under the curve was calculated to be 0.9447 with a 95\% confidence interval of 0.8943-0.9960 ( $\mathrm{P}<0.0001$; Fig. 2B), suggesting that TUG1 expression can be used to diagnose osteosarcoma effectively.

Factors affecting TUG1 expression and prognostic value of TUG1 expression for osteosarcoma. Patients with osteosarcoma were divided into two groups according to the median expression levels of TUG1 in plasma. LncRNA expression 
A

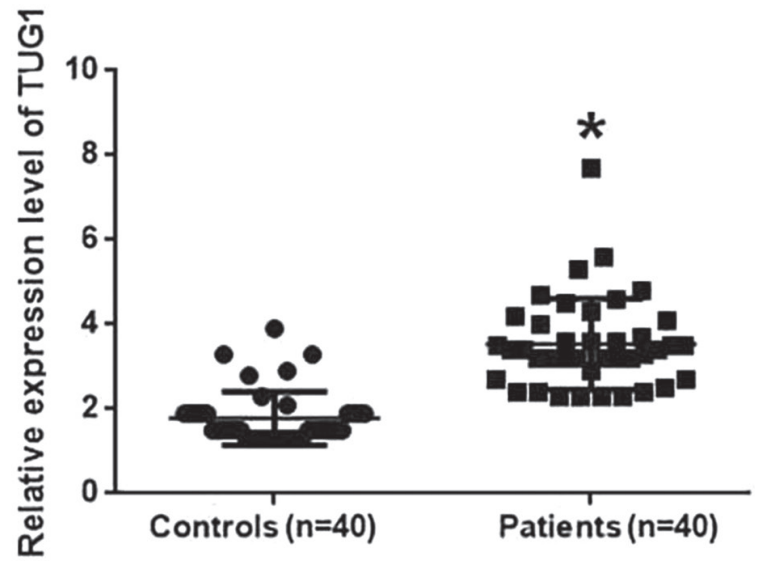

B

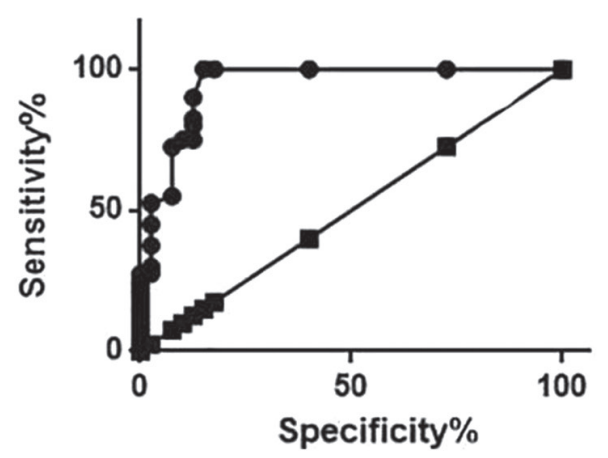

Figure 2. Relative expression levels of lncRNA TUG1 in the plasma samples of osteosarcoma patients and healthy controls, and their diagnostic value for osteosarcoma. (A) Relative expression levels of lncRNA TUG1 in the plasma of osteosarcoma patients and healthy controls. (B) Diagnostic value of TUG1 for osteosarcoma. "P<0.05 vs. Controls. LncRNA, long non-coding RNA; TUG1, taurine upregulated gene 1.

has been found to be dependent on lifestyle factors, including smoking and drinking (11). In the present study, the expression level of TUG1 was not associated with sex, age, or history of drinking or smoking, but was associated significantly with tumor metastasis $(\mathrm{P}<0.001$; Table I), suggesting that TUG1 is a valid diagnostic marker for osteosarcoma. The Kaplan-Meier method was next applied to produce a survival curve. Overall patient survival in the group with high TUG1 expression was found to be significantly lower compared with that in patients with low TUG1 expression $(\mathrm{P}<0.005$; Fig. 3$)$. These results suggest that TUG1 can serve as a prognostic marker for osteosarcoma.

Effects of TUG1 knockdown by siRNA and vector-induced overexpression on osteosarcoma cell viability. The expression levels of TUG1 were found to be significantly lower in the hFOB human normal bone cell line compared with those in the MG-63 and U2OS osteosarcoma cell lines $(\mathrm{P}<0.05$; Fig. 4A). According to RT-qPCR results, transfection with plasmid expressing TUG1 significantly increased TUG1 expression ( $\mathrm{P}<0.05$; Fig. 4B), whereas transfection with TUG1 siRNA significantly reduced TUG1 expression in MG-63 and U2OS cells ( $\mathrm{P}<0.05$; Fig. 4C), demonstrating that the transfection was efficient. TUG1 overexpression significantly increased MG-63 and U2OS cell viability (P<0.05; Fig. 4D), while TUG1 knockdown significantly reduced cell viability $(\mathrm{P}<0.05$; Fig. 4E). These data suggest that the expression level of TUG1 is positively associated with viability in osteosarcoma cells.

Effects of TUG1 silencing and overexpression on osteosarcoma cell migration and invasion. Transwell migration and invasion assays were performed to investigate the effects of changes in TUG1 expression on cell migration and invasion. TUG1 overexpression significantly increased MG-63 and U2OS cell migration and invasion ( $\mathrm{P}<0.05$; Fig. $5 \mathrm{~A}$ and $\mathrm{B})$. By contrast, TUG1 knockdown significantly reduced the migratory and invasive capabilities of the same cell lines $(\mathrm{P}<0.05$; Fig. 5C and D). Those observations suggest that expression level of TUG1 is positively associated with the migration and invasion abilities of osteosarcoma cells.

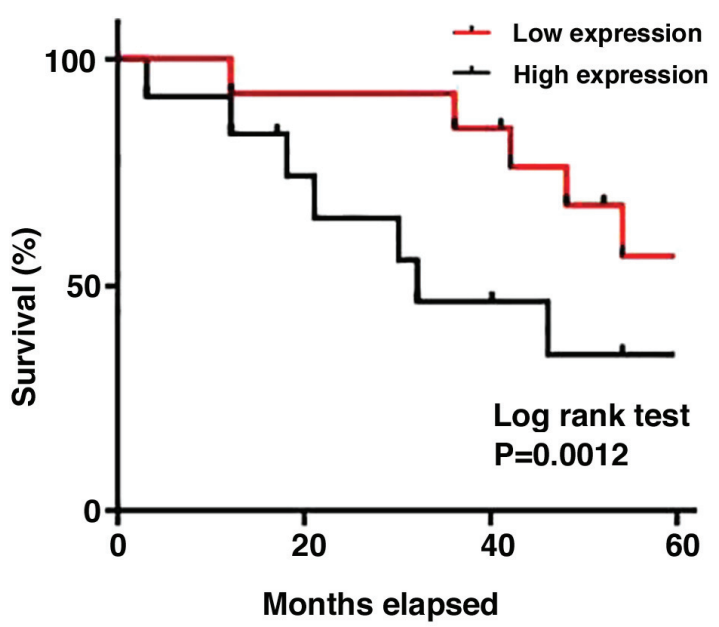

Figure 3. Kaplan-Meier survival curve of patients with high and low expression of taurine-upregulated gene 1 .

TUG1 overexpression upregulates RUNX2 expression in osteosarcoma cells. The RUNX2 protein expression levels of MG-63 and U2OS cells overexpressing TUG1 were evaluated using western blot analysis. Compared with un-transfected control and negative control cells transfected with empty vectors, IncRNA TUG1 overexpression resulted in significantly upregulated RUNX2 expression in both cell lines $(\mathrm{P}<0.05$; Fig. 6).

\section{Discussion}

Osteosarcoma is a rare but devastating disease. Although genetic factors, including tumor protein P53 and retinoblastoma tumor suppressor gene, have been demonstrated to be involved in the development of osteosarcoma, the pathogenesis of this disease remains poorly characterized $(12,13)$. LncRNA is a group of functional RNAs that do not encode proteins. Despite the lack of protein-coding ability, lncRNAs have been shown serve a role in almost every aspect of critical biological and pathological processes (14). Previous studies have found that the development of osteosarcoma is closely associated with 
A
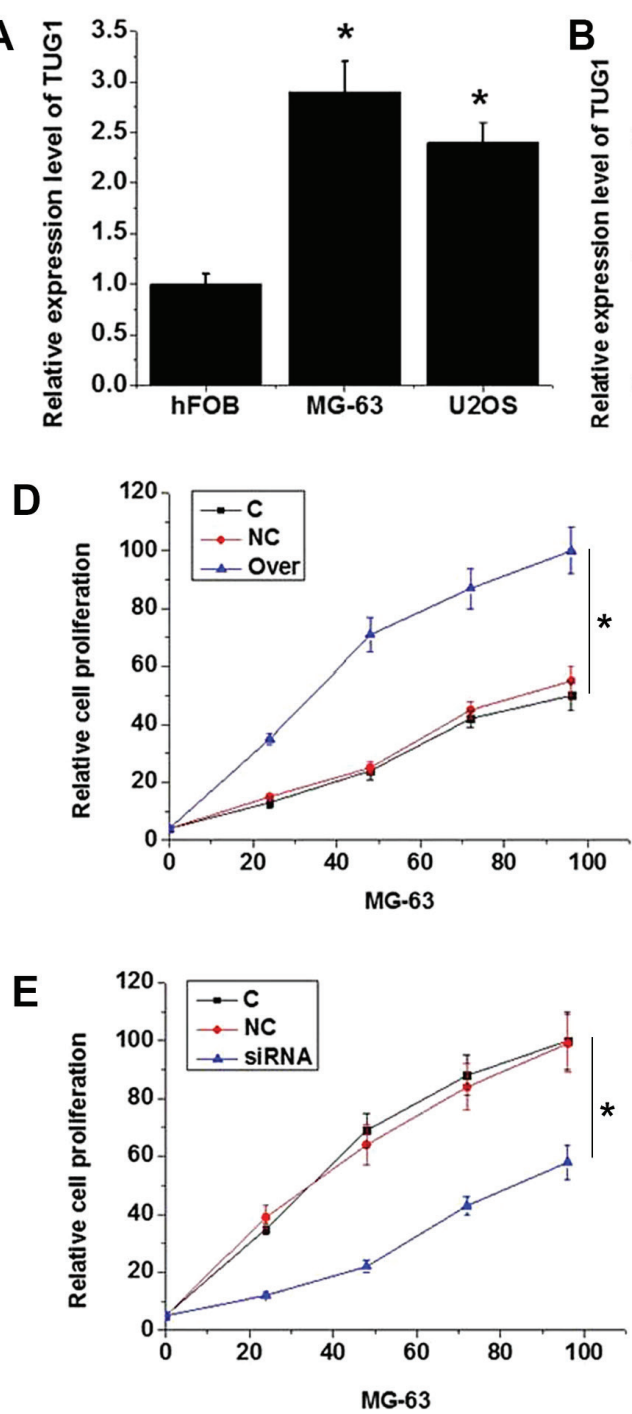
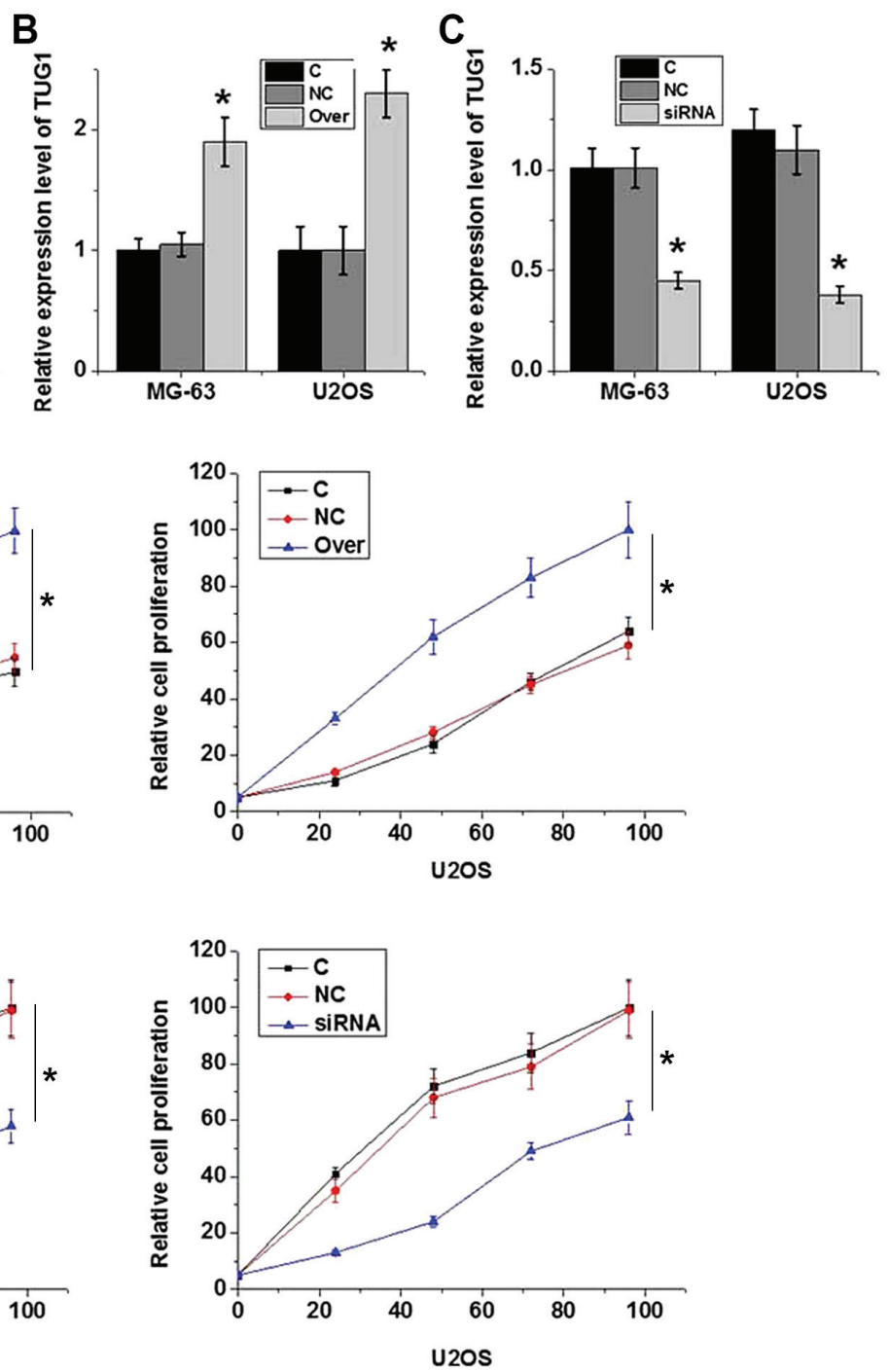

Figure 4. Effects of TUG1 knockdown and overexpression on cell viability. (A) Relative TUG1 expression in normal human bone cell line hFOB and osteosarcoma cell lines MG-63 and U2OS. "P<0.05 vs. hFOB. (B) Relative TUG1 expression in MG-63 and U2OS cells following transfection with plasmid expressing TUG1. "P<0.05 vs. C and NC. (C) Relative TUG1 expression in MG-63 and U2OS cells following transfection with NC or TUG1 siRNA. "P<0.05 vs. C and NC. (D) Effects of TUG1 overexpression on MG-63 and U2OS cell viability. (E) Effects of TUG1 knockdown on MG-63 and U2OS cell viability. "P<0.05 vs. C and NC. Each experiment was repeated three times. For cell viability experiments, all data were normalized to the control group at $96 \mathrm{~h}$. TUG1, taurine upregulated gene 1; siRNA, small interfering RNA; Over, cells transfected with plasmid expressing TUG1; C, controls cells without any transfection; NC, negative control cells transfected with empty vector or negative control siRNA.

the abnormal expression of a number of lncRNAs $(9,15,16)$. In particular, high levels of lncRNA TUG1 have been shown to be associated with poor prognosis in patients with osteosarcoma (9). In osteosarcoma cell lines, lncRNA TUG1 expression is upregulated compared with that in normal osteoblastic cells, and the overexpression of IncRNA TUG1 promotes osteosarcoma cell proliferation (15). By contrast, the downregulation of lncRNA TUG1 has been shown to reduce osteosarcoma cell proliferation and increase cancer cell apoptosis (16). In addition, an association of the upregulation of lncRNA TUG1 with the poor survival of osteosarcoma patients has been reported (9). Consistent with these findings from previous studies, the present study revealed that the expression levels of TUG1 were significantly higher in osteosarcoma tissues compared with adjacent healthy tissues. TUG1 levels were also demonstrated to be higher in the plasma samples of osteosarcoma patients compared with those from healthy controls.
Taken together, this suggests that TUG1 may serve a role as an oncogene in osteosarcoma.

The pathogenesis of certain human malignancies is frequently accompanied by changes in biological molecules such as lncRNA in the plasma (17). Notably, plasma lncRNA has been widely applied in cancer diagnosis; a recent study reported that the plasma levels of IncRNA SOX2-OT were significantly increased in patients with osteosarcoma compared with healthy controls, which indicated poor prognosis (18). Although lncRNA TUG1 has been reported to be involved in the development of osteosarcoma, its diagnostic and prognostic potential for osteosarcoma remain unreported. In the present study, ROC curve analysis showed that increased levels of TUG1 expression could be used effectively to predict osteosarcoma. In addition, survival times of patients with higher levels of TUG1 expression were significantly shorter compared with those of patients with lower expression levels of TUG1, further 
A
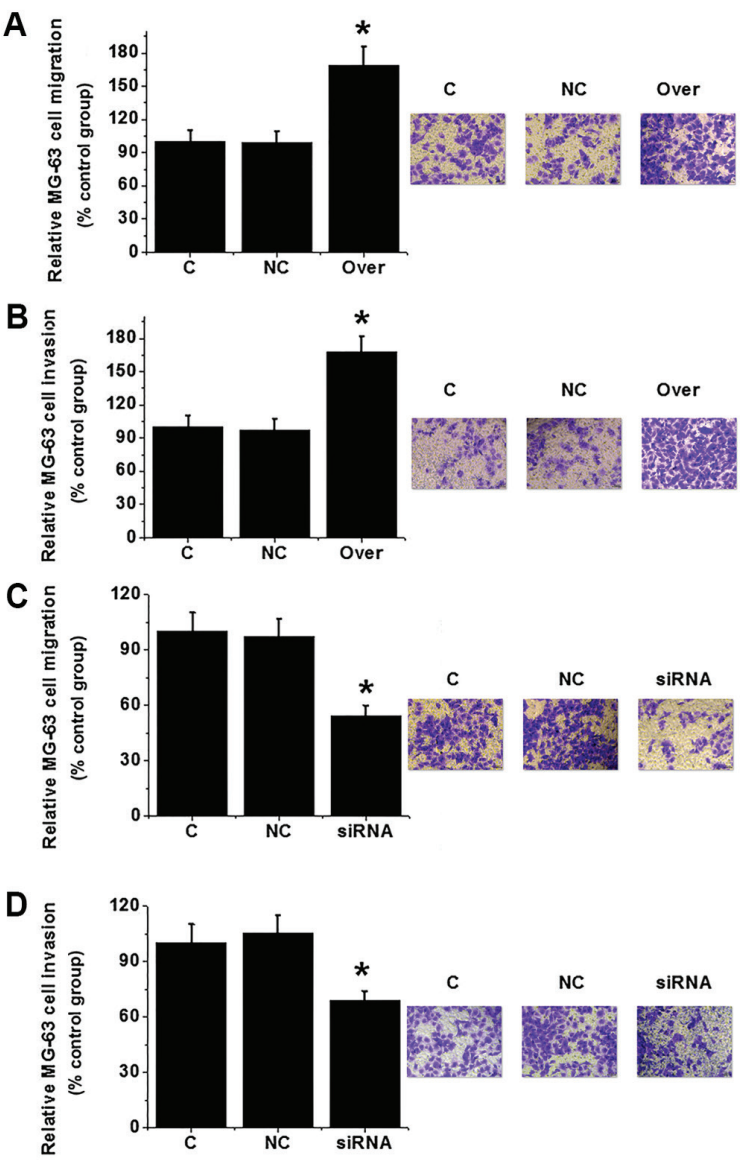
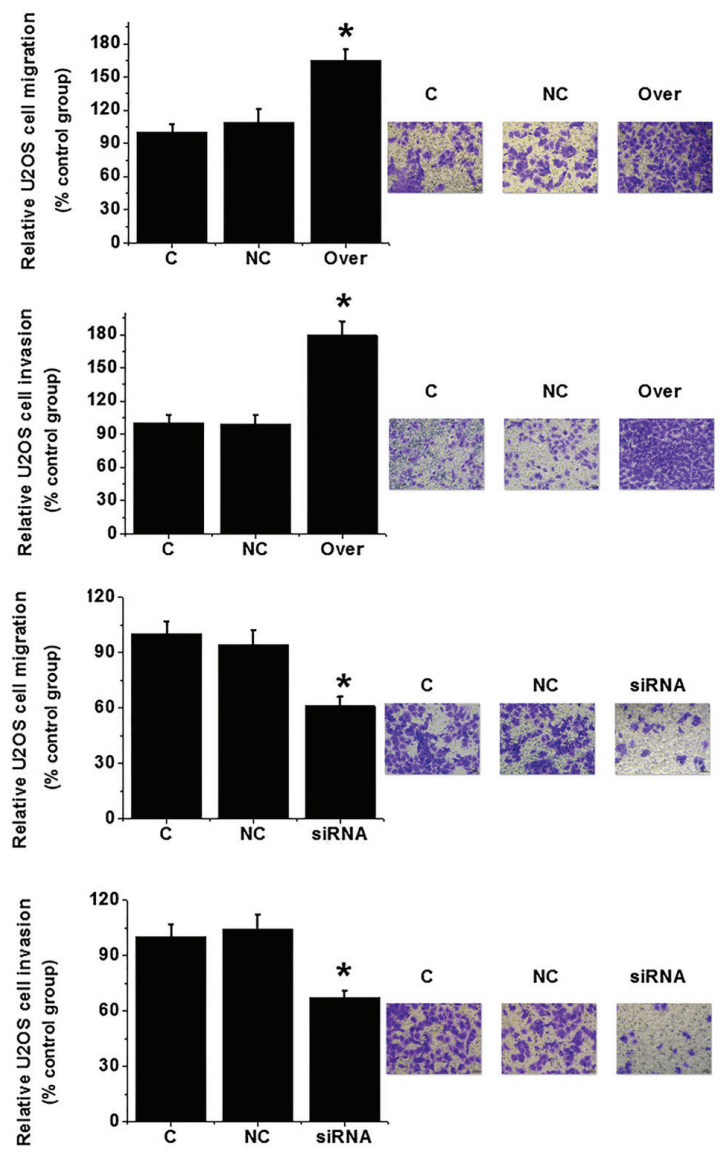

Figure 5. Effects of TUG1 knockdown and overexpression on cell migration and invasion. (A) Migration and (B) invasion of MG-63 and U2OS cells following TUG1 overexpression. (C) Migration and (D) invasion of MG-63 and U2OS cells following TUG1 knockdown. Each experiment was repeated three times, and all data were normalized to $\mathrm{C}$. ${ }^{*} \mathrm{P}<0.05$ vs. $\mathrm{C}$ and NC. TUG1, taurine upregulated gene 1 ; $\mathrm{C}$, control cells without any transfection; NC, negative control cells transfected with empty vector or negative control siRNA; Over, cells transfected with plasmid expressing TUG1.
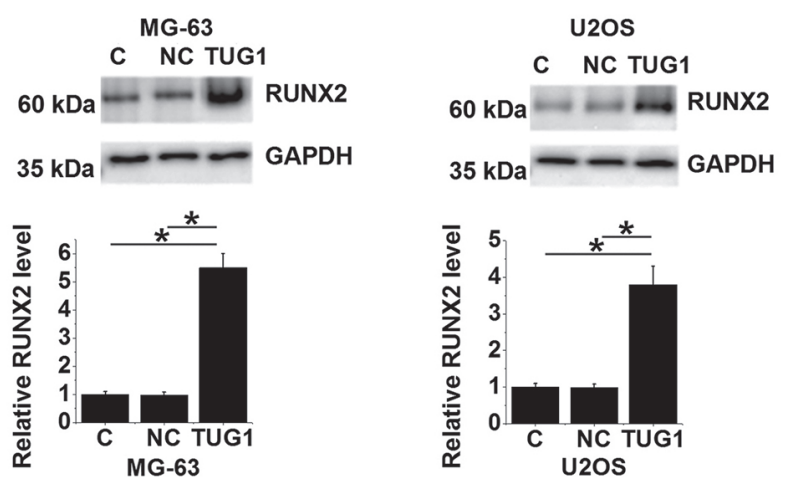

Figure 6. LncRNA TUG1 expression correlates positively with RUNX2 expression in osteosarcoma cells. Western blot analysis of RUNX2 in MG-63 and U2OS cells following lncRNA TUG1 overexpression. ${ }^{*} \mathrm{P}<0.05$ vs. NC and C. LncRNA, long non-coding RNA; TUG1, taurine upregulated gene 1; RUNX2, Runt-related transcription factor 2; C, controls cells without any transfection; NC, negative control cells transfected with empty vectors.

supporting the significant diagnostic and prognostic values of TUG1 expression for osteosarcoma. It is well accepted that the expression of some lncRNAs can be influenced by lifestyle factors, including drinking and smoking (11). In the present study, the levels of TUG1 expression did not associate significantly with age, sex, or history of drinking or smoking among patients with osteosarcoma. Instead, TUG1 expression levels were found to be associated significantly with tumor metastasis. These data suggest serum TUG1 expression to be a promising biomarker for osteosarcoma.

TUG1 may participate in the development of a number of human malignancies by regulating cancer cell proliferation, migration and invasion $(9,15,16)$. Zhang et al (19) found that the downregulation of TUG1 significantly inhibited proliferation, migration and invasion but promoted apoptosis in renal cell carcinoma cells, suggesting that TUG1 is a promising target for this disease. In addition, downregulation of TUG1 was also found to inhibit proliferation and promote cell apoptosis in osteosarcoma cells (20). In the present study, TUG1 overexpression significantly increased osteosarcoma cell viability, migration and invasion, respectively, while siRNA-mediated TUG1 knockdown and significantly suppressed them. EMT is one of the key steps for cancer cell migration and invasion (21). RUNX2 contributes to the growth and metastasis of osteosarcoma (22). In the present study, expression levels of RUNX2 mRNA positively correlated with those of lncRNA TUG1 in tumor tissues but not in adjacent healthy tissues. LncRNA TUG1 overexpression led to significantly upregulated RUNX2 in osteosarcoma cell lines. These findings suggest that TUG1 can upregulate RUNX2 to promote osteosarcoma cell migration and invasion.

Due to the limited number of young participants, the average age of the research subjects in the present study 
was $41 \pm 11.1$ years, which does not reflect the general demographics of this disease and, therefore, serves as a limitation. In future studies analysis including samples from younger patients is necessary to confirm the conclusions. In addition, this present study lacks in vivo experimental data. Experiments using animal models should also be included in any future studies.

In conclusion, the expression levels of TUG1 were found to be significantly higher in osteosarcoma tissues compared with adjacent healthy tissues, and to be significantly higher in the plasma samples of osteosarcoma patients compared with healthy controls. This suggests that TUG1 expression has significant diagnostic and prognostic value for osteosarcoma. Mechanistically, TUG1 likely promotes osteosarcoma cell proliferation, migration and invasion by upregulating RUNX2.

\section{Acknowledgements}

Not applicable.

\section{Funding}

No funding was received.

\section{Availability of data and materials}

The datasets used and/or analyzed during the current study are available from the corresponding author on reasonable request.

\section{Authors' contributions}

KS designed experiments. KS and YL performed experiments and analyzed data. KS drafted the paper and both authors approved the paper.

\section{Ethics approval and consent to participate}

This study was approved by the ethics committee of The 2nd Affiliated Hospital of Zhejiang University School of Medicine, and all patients provided signed informed consent.

\section{Patient consent for publication}

Not applicable.

\section{Competing interests}

The authors declare that they have no competing interests.

\section{References}

1. Durfee RA, Mohammed M and Luu HH: Review of osteosarcoma and current management. Rheumatol Ther 3: 221-243, 2016.

2. Lindsey BA, Markel JE and Kleinerman ES: Osteosarcoma overview. Rheumatol Ther 4: 25-43, 2017.

3. Lin YH, Jewell BE, Gingold J, Lu L, Zhao R, Wang LL and Lee DF: Osteosarcoma: Molecular pathogenesis and iPSC modeling. Trends Mol Med 23: 737-755, 2017.
4. Isakoff MS, Bielack SS, Meltzer P and Gorlick R: Osteosarcoma: Current treatment and a collaborative pathway to success. J Clin Oncol 33: 3029-3035, 2015.

5. Esteller M: Non-coding RNAs in human disease. Nat Rev Genet 12: 861-874, 2011.

6. Perkel JM: Visiting 'noncodarnia'. Biotechniques 54: 301, 303-304, 2013.

7. Li J, Zhang M, An G and Ma Q: LncRNA TUG1 acts as a tumor suppressor in human glioma by promoting cell apoptosis. Exp Biol Med (Maywood) 241: 644-649, 2016.

8. Xu Y, Wang J, Qiu M, Xu L, Li M, Jiang F, Yin R and Xu L: Upregulation of the long noncoding RNA TUG1 promotes proliferation and migration of esophageal squamous cell carcinoma. Tumor Biol 36: 1643-1651, 2015.

9. Ma B, Li M, Zhang L, Huang M, Lei JB, Fu GH, Liu CX, Lai QW, Chen QQ and Wang YL: Upregulation of long non-coding RNA TUG1 correlates with poor prognosis and disease status in osteosarcoma. Tumor Biol 37: 4445-4455, 2016.

10. Livak KJ and Schmittgen TD: Analysis of relative gene expression data using real-time quantitative PCR and the 2(-Delta Delta C(T)) method. Methods 25: 402-408, 2001.

11. Thai P, Statt S, Chen CH, Liang E, Campbell $\mathrm{C}$ and $\mathrm{Wu} \mathrm{R}$ : Characterization of a novel long noncoding RNA, SCAL1, induced by cigarette smoke and elevated in lung cancer cell lines. Am J Respir Cell Mol Biol 49: 204-211, 2013.

12. Wang L, Zhao Z, Feng W, Ye Z, Dai W, Zhang C, Peng J and Wu K: Long non-coding RNA TUG1 promotes colorectal cancer metastasis via EMT pathway. Oncotarget 7: 51713-51719, 2016.

13. Kansara M and Thomas DM: Molecular pathogenesis of osteosarcoma. DNA Cell Biol 26: 1-18, 2007.

14. Chen X and Yan GY: Novel human lncRNA-disease association inference based on lncRNA expression profiles. Bioinformatics 29: 2617-2624, 2013.

15. Yun-Bo F, Xiao-Po L, Xiao-Li L, Guo-Long C, Pei Z and Fa-Ming T: LncRNA TUG1 is upregulated and promotes cell proliferation in osteosarcoma. Open Med (Wars) 11: 163-167, 2016.

16. Zhang Q, Geng PL, Yin P, Wang XL, Jia JP and Yao J: Down-regulation of long non-coding RNA TUG1 inhibits osteosarcoma cell proliferation and promotes apoptosis. Asian Pac J Cancer Prev 14: 2311-2315, 2013.

17. Hori SS, Lutz AM, Paulmurugan R and Gambhir SS: Correlation of plasma biomarker levels with early-stage tumor viability in an orthotopic ovarian cancer mouse model. Cancer Res 74 (19 Suppl): Abstract nr 873, 2014.

18. Wang Z, Tan M, Chen G, Li Z and Lu X: LncRNA SOX2-OT is a novel prognostic biomarker for osteosarcoma patients and regulates osteosarcoma cells proliferation and motility through modulating SOX2. IUBMB Life 69: 867-876, 2017.

19. Zhang M, Lu W, Huang Y, Shi J, Wu X, Zhang X, Jiang R, Cai Z and Wu S: Downregulation of the long noncoding RNA TUG1 inhibits the proliferation, migration, invasion and promotes apoptosis of renal cell carcinoma. J Mol Histol 47: 421-428, 2016.

20. Zhang Q, Geng PL, Yin P, Wang XL, Jia JP and Yao J: Down-regulation of long non-coding RNA TUG1 inhibits osteosarcoma cell proliferation and promotes apoptosis. Asian Pac J Cancer Prev 14: 2311-2315, 2013.

21. Rokavec M, Öner MG, Li H, Jackstadt R, Jiang L, Lodygin D, Kaller M, Horst D, Ziegler PK, Schwitalla S, et al: IL-6R/STAT3/miR-34a feedback loop promotes EMT-mediated colorectal cancer invasion and metastasis. J Clin Invest 124: 1853-1867, 2014.

22. Martin JW, Zielenska M, Stein GS, van Wijnen AJ and Squire JA: The role of RUNX2 in osteosarcoma oncogenesis. Sarcoma 2011: 282745, 2011.

This work is licensed under a Creative Commons Attribution-NonCommercial-NoDerivatives 4.0 International (CC BY-NC-ND 4.0) License. 